\title{
Lösungsmöglichkeiten bei fehlgeschlagenen Osteosynthesen an der Hand
}

Martin F. Langer, Michael J. Raschke

\author{
Wann ist eine Osteosynthese an der Hand fehlgeschlagen? Auf der Suche nach einer \\ Definition für eine fehlgeschlagene Osteosynthese an der Hand - für die es unzählige \\ unterschiedliche Möglichkeiten gibt - sind wir zu dem ganz einfachen Schluss ge- \\ kommen, dass eine Osteosynthese dann fehlgeschlagen ist, wenn revidiert werden \\ muss. Revidiert werden muss, wenn das Ziel der Knochenheilung in anatomischer \\ Position ohne Beeinträchtigung der umgebenden funktionellen Strukturen und ohne \\ verbleibende Schmerzen in annehmbarer Zeit nicht erreicht werden kann.
}

Bevor man zur Revisionsoperation schreitet, muss eine intensive Fehleranalyse gemacht werden. Warum ist die Osteosynthese fehlgeschlagen? Zuerst muss sich jeder Chirurg fragen, was habe ich oder was wurde von ärztlicher Seite falsch gemacht, bevor das Osteosynthesematerial, die Knochenqualität, der Patient oder der Physiotherapeut verantwortlich gemacht wird.

„Dem Chirurgen wird ein schlechter Ausgang in höherem Sinne zur persönlichen Schuld. Tragbar wird diese Belastung durch Gewissenhaftigkeit in der Indikationsstellung, Beherrschung der Technik und ein berechtigtes Selbstbewusstsein. Seine sicherste Stütze aber ist die Wahrhaftigkeit. Der Chirurg, der deutelt, Fehlschläge zu entschuldigen sucht, verstößt gegen das vornehmste Gesetz seiner Zunft."

\section{Ferdinand Sauerbruch (1875-1951)}

Was wurde nicht berücksichtigt? Wurde das Implantat falsch platziert oder zu schwaches Material eingesetzt? Wurde nicht richtig gemessen? War der Zugangsweg falsch? Ist das Implantat zu nah am Gelenk, sodass die Kapsel blockiert wird? Wurden die Fragmente suffizient gefasst? Wurde zu lange gewartet? Und vieles mehr. Aber nicht alle fehlgeschlagenen Osteosynthesen lassen sich vermeiden. Es gibt zahlreiche Situationen, die sich erst sekundär trotz erstklassiger Osteosynthese entwickeln. Diese Fehleranalyse ist wichtig, um diesen oder ähnliche Fehler bei der Revision und auch bei primären Operationen nicht zu wiederholen.

Osteosynthesen an der Hand müssen in vielfacher Hinsicht anders bewertet werden als an anderen Körperstellen. Kaum ein anderer Knochen hat eine so hohe Pseudarthrosenrate wie das Kahnbein und benötigt eine atraumatische, sehr exakte Reposition und Schraubenosteo- synthese mit anschließender Ruhigstellung. Die Handwurzel ist aber schon fast der einzige Bereich, an dem eine längere Ruhigstellung erlaubt ist. Frakturen an allen anderen Regionen der Hand sind extrem gefährdet für Sehnen- und Kapselverklebungen. Man muss eine Osteosynthese auch dann als fehlgeschlagen bezeichnen, wenn trotz intensiver Übungsbehandlung deutliche Bewegungsstörungen an der Hand entstehen. Zur Vermeidung von steifen und kontrakten Fingern ist eine sehr frühzeitige Übungsbehandlung notwendig. Die notwendigerweise zarten Osteosynthesen mit kleinsten Drähten, Schrauben und Platten müssen den biomechanischen Belastungen einer frühen Übung standhalten. Die Zugangswege an der Hand, die ein Sinnesorgan darstellt, sind aufgrund der zu schonenden Nerven, Gefäße und Sehnen immer speziell.

Grundsätzlich muss man diese Revisionsoperationen nach dem Zeitpunkt der Revision einteilen: So gibt es Situationen, die zur sofortigen Revision zwingen (Frührevision), und Situationen, die nach einigen Wochen, einigen Monaten oder Jahren zur Spätrevision zwingen. Sofortige Revisionen sind grobe Fehllagen des Osteosynthesematerials, instabile Versorgungen oder Fehlstellungen der Knochen, die biomechanisch/funktionell nicht akzeptabel sind. Im günstigsten Fall fällt die fehlgeschlagene Osteosynthese noch intraoperativ auf, z. B. bei der intraoperativen Durchleuchtung oder beim Belastungsund Provokationstest. Dann kann sofort bei noch offenen Wunden eine Lösung gefunden werden. Spätrevisionen kommen bei Auslockerungen, Materialermüdungen mit Draht-, Schrauben- oder Plattenbruch vor, bei Implantatentfernungen aufgrund von Sehnenverklebungen, Pseudarthrosen oder Kapselkontrakturen. 
Bevor man zum Messer greift, sollte man sehr gut vorbereitet sein. Eine ausführliche klinische und apparative Diagnostik, z.B. durch Computertomografie, ist hier noch wichtiger als beim Ersteingriff. Falls nötig, ist auch eine präoperative Handtherapie sinnvoll, damit die Beweglichkeit der nicht direkt betroffenen Gelenke so gut es geht verbessert wird und sofort postoperativ beim bekannten Handtherapeuten die intensiven Bewegungsübungen fortgesetzt werden können. Der Chirurg muss einen klaren Plan für die Revisionsoperation haben, die notwendigen Revisionsimplantate sowie mehrere Ausweichmöglichkeiten (Rettungsoperationen) parat haben, falls die geplante Revisionstaktik nicht möglich ist oder es sich intraoperativ herausstellen sollte, dass dieser Plan nicht sinnvoll ist. Über alle Möglichkeiten und Ausweichmöglichkeiten muss der Patient vor der Operation aufgeklärt werden und die speziellen Wünsche oder Erfordernisse des Patienten (Beruf - Hobby) müssen bekannt sein. Vor allem aber braucht der Operateur viel Kreativität.

\section{Fehlgeschlagene Osteosynthesen am Kahnbein}

Skaphoidfrakturen sind ausgesprochen häufig und werden heute, wenn sie frühzeitig erkannt werden, mit Kahnbeinschrauben versorgt. Diese ursprünglich von Timothy Herbert entwickelten Doppelgewindeschrauben sind heute fast immer kanüliert und werden über vorgebohrte Kirschner-Drähte eingebracht. Aus praktischen Gründen ist die Richtung der Verschraubung bei den häufigen Querfrakturen meist retrograd von palmar und bei kleinen proximalen Fragmenten anterograd von dorsal. Zweifellos braucht der Operateur für die Einbringung der Schraube viel Übung, denn das Kahnbein ist nicht gerade, sondern mehrfach in sich gedreht aufgebaut (Langer) [1]. Da die Skaphoidfragmente nicht selten gegeneinander verdreht sind - u.a. die sog. Humpback-Deformität kann es vorkommen, dass diese Fragmente in Fehlposition, unter Verkürzung oder ohne gute Kontaktflächen zueinander verschraubt werden. Durch die „perkutane“ Technik können die Schrauben Fragmente verfehlen und die intraoperativen Durchleuchtungsbilder sind manchmal schwer zu interpretieren. Erst ein postoperatives CT zeigt die wahren Verhältnisse. Wenn die Skaphoidschraube nicht gut platziert oder zu kurz ist, die Fragmente nicht gut reponiert sind und zu wenig unter Kompression stehen sowie zu viel Bewegung im Bereich der Handwurzel vor der Knochenheilung herrscht, kann auch bei liegender Schraube eine Pseudarthrose entstehen. Das Problem ist dann, dass um die Kahnbeinschraube ein breiter Resorptionssaum besteht und es kaum möglich ist, eine neue Schraube stabil zu platzieren. Zu lange Schrauben können entweder am Radius, im STT-Gelenk (STT: Skaphoid-Trapezium-Trapezoideum-Gelenk) oder an den Weichteilen Probleme bereiten.
Wenn bei den postoperativen Röntgenbildern Zweifel über eine korrekte Lage der Kahnbeinschraube bestehen, sollte ein CT durchgeführt werden. Ebenso ist ein CT notwendig, wenn es nach ausreichender Zeit zu keiner Heilung oder erneut zu einer Pseudarthrose bei liegender Schraube kommt. Für eine Revisionsoperation am Kahnbein ist die Information aus dem CT unbedingt notwendig. Über eine Revisionsoperation muss nachgedacht werden,

1. wenn die Osteosynthese die Fragmente nicht ausreichend fasst,

2. eine Fehlstellung noch besteht oder sich entwickelt hat, die eine Heilung nicht erwarten lässt,

3. eine so deutliche Fehlstellung besteht, dass nach Heilung Probleme zu erwarten sind, oder

4. nach einer Osteosynthese schmerzhafte Handgelenksbeschwerden bestehen.

Die häufigsten Szenarien mit deren Lösungsmöglichkeiten für das Kahnbein werden im Folgenden dargestellt.

\section{Szenario 1: Frische Versorgung mit einer fehl- platzierten, zu kurzen oder zu langen Schraube}

Die fehlerhafte Schraube muss auf jeden Fall entfernt werden. Dazu muss der vorgegebene Zugang wieder eröffnet und das proximale Ende der Schraube freigelegt werden. Dies ist nicht immer einfach, da die Schrauben manchmal tief im Skaphoid versenkt sind. Wenn die Erstoperation noch nicht zu lange zurückliegt, kann mit einem passenden Kirschner-Draht der Kanal ausgetastet und der Draht in die kanülierte Schraube eingeführt werden ( $\triangleright$ Abb. 1). Dies erleichtert das Finden und den Zugang zur Schraube in der Tiefe, deren Verbindung zum Schraubendreher sehr sauber freigelegt werden muss. Der genaue Schraubentyp muss bekannt sein, um den genau passenden Schraubendreher einsetzen zu können, was sehr wichtig ist. Denn die Schraube kann schon fest eingemauert sein. Bevor man die erste Umdrehung mit dem Schraubendreher macht, muss also ganz klar sein, dass der richtige Schraubendreher den vollen Kontakt zum Schraubenansatz hat. Narbengewebe oder Knochenreste zwischen Schraubendreher und Schraube führen zum Abrutschen des Schraubendrehers und ggf. zur Zerstörung des heute gebräuchlichen Inbus- oder TorxAnsatzes der Schraube. Was passiert, wenn die Schraube mit dem Schraubendreher nicht entfernt werden kann, kann sich jeder leicht vorstellen. Feine Meißel, feine scharfe Löffel und feine Klemmen sollten dann bereitstehen.

Wenn die Schraube entfernt ist, muss abgeschätzt werden, ob die Knochensubstanz es zulässt, dass ohne weitere Auffüllung eine andere Schraube an der richtigen Position und mit der richtigen Länge eingebracht werden kann. Manchmal ist auch ein Wechsel von der Beugeseite zur Streckseite zum Einbringen der Schraube hilfreich, da auf diese Weise ganz andere Richtungen eingeschlagen 

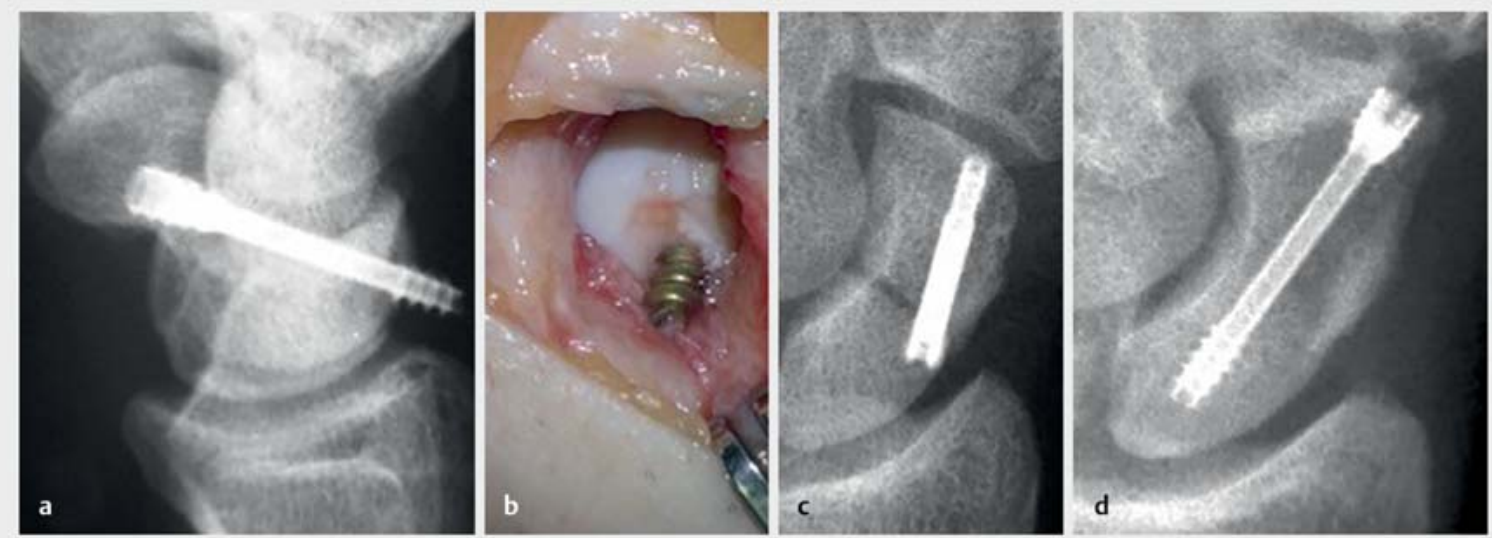

Abb. 1 Fehlplatzierte Skaphoidschrauben: a, b Skaphoidschraube zu flach eingebracht, sodass sie dorsal weit austritt. c, d Proximales Fragment wurde über dorsalen Zugang nicht ausreichend von Skaphoidschraube gefasst (c), Revisionsoperation von palmar mit guter Kompression.
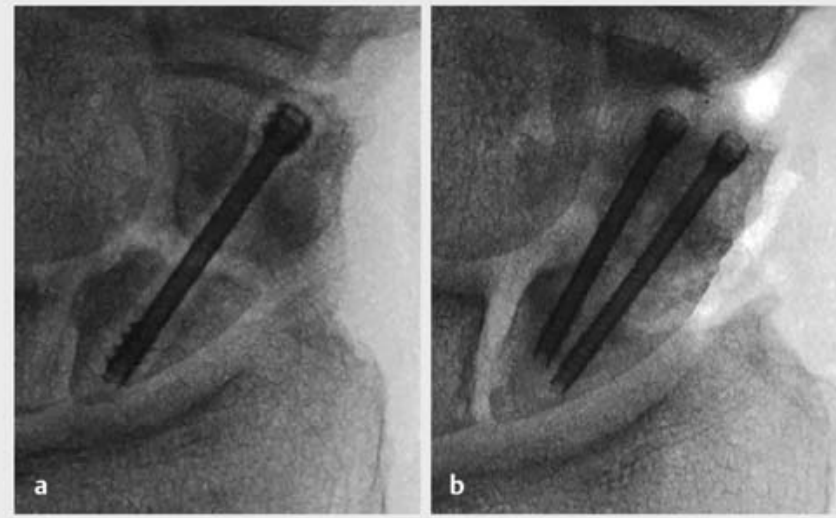

- Abb. 2 Ausgelockerte Skaphoidschraube: a Lockerungssaum um die Skaphoidschraube gut zu erkennen. b Nach Spongiosaauffüllung Stabilisierung mit 2 2,2-mm-Skaphoidschrauben.

werden können. Eine anatomische Reposition ist dabei immer Voraussetzung. Ansonsten sollte der alte Schraubenkanal mit Spongiosa aus dem distalen Radius aufgefüllt werden.

\section{Szenario 2: Lange zurückliegende Versorgung mit ausgelockerter, sonst korrekt liegender Schraube und Pseudarthrose}

Die Schraube lässt sich in diesem Fall meist leicht entfernen, die Chance für eine stabile Neuplatzierung einer anderen Schraube ist aber gering, da der Hohlraum um die Schraube größer geworden ist und zusätzlich meist eine deutliche Humpback-Deformität vorliegt ( $\bullet$ Abb. 2). Eine
Beckenkammspongiosaplastik ist hier häufig unumgänglich. Statt einer normalen Skaphoidschraube können auch 2 dünne Skaphoidschrauben eingebracht werden. Zusätzlich ist ein kortikospongiöser nagelartiger Span sinnvoll, um die vergrößerte Schraubenhöhle dübelartig (siehe $>$ Abb. 3) aufzufüllen. Ansonsten brechen die neuplatzierten Schrauben in diese Höhle ein. Alleinige Spongiosa würde nicht genug Halt geben [2, 3].

\section{Szenario 3: Schraube lockert aus, da der nach einer Pseudarthrosenoperation eingesetzte Span nicht einheilt oder teilweise nekrotisch wird}

Der Defekt im Kahnbein ist nach Aufrichtung und Anfrischung hier meist so groß, dass einerseits sehr große Mengen an kortikospongiösen Knochenanteilen eingebracht werden müssen und andererseits kaum noch stabile Anteile des Restkahnbeins vorhanden sind, in die eine Schraube eingebracht werden könnten. Hier sind entweder mehrere Kirschner-Drähte (K-Drähte) oder eine Kahnbeinplatte [4] die einzigen Möglichkeiten zur Osteosynthese ( $\triangleright$ Abb. 4). Ist der Defekt noch größer oder ist der proximale Pol betroffen, so kommen heute entweder vaskularisierte Knochenspäne [5] oder verschiedene Rettungsoperationen (Proximal Row Carpectomy; mediokarpale Teilarthrodese) in Betracht ( $\triangleright \mathbf{A b b} \mathbf{5} \mathbf{a}$ und $\mathbf{b}$ ).

\section{Szenario 4: Skaphoid in Fehlstellung verheilt und es kommt zu zunehmenden Arthrosen bzw. zum karpalen Kollaps}

Bei Arthrosen [6] der radioskaphoidalen Gelenkfläche oder bei einem weiter fortgeschrittenen karpalen Kollaps bieten sich, je nach Handgelenksbefund, verschiedene Rettungsoperationen an: Ist neben der Fovea scaphoidea auch die Fovea lunata arthrotisch verändert, kann eine ra- 

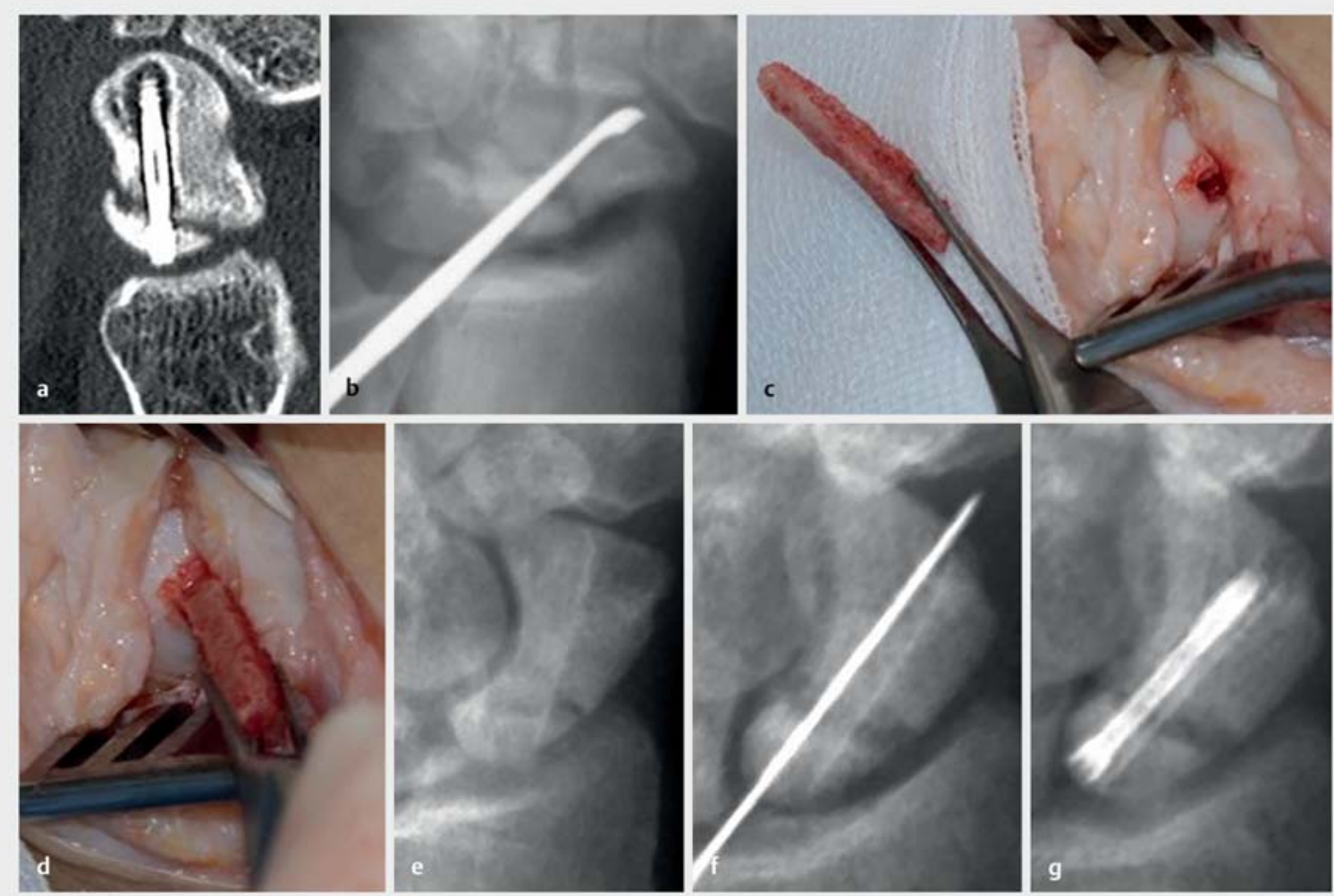

- Abb. 3 Weit ausgelockerte Skaphoidschraube: a CT der weit ausgelockerten Schraube. b Auskratzen des Schraubenkanals mit einem kleinen scharfen Löffel. c Dübelartiger kortikospongiöser Beckenkammspan. d Einschlagen des „Knochendübels“. e Kanalauffüllung mit Spongiosa und Knochendübel. f Kirschner-Draht-Vorbohrung. g Besetzen mit neuer Skaphoidschraube.
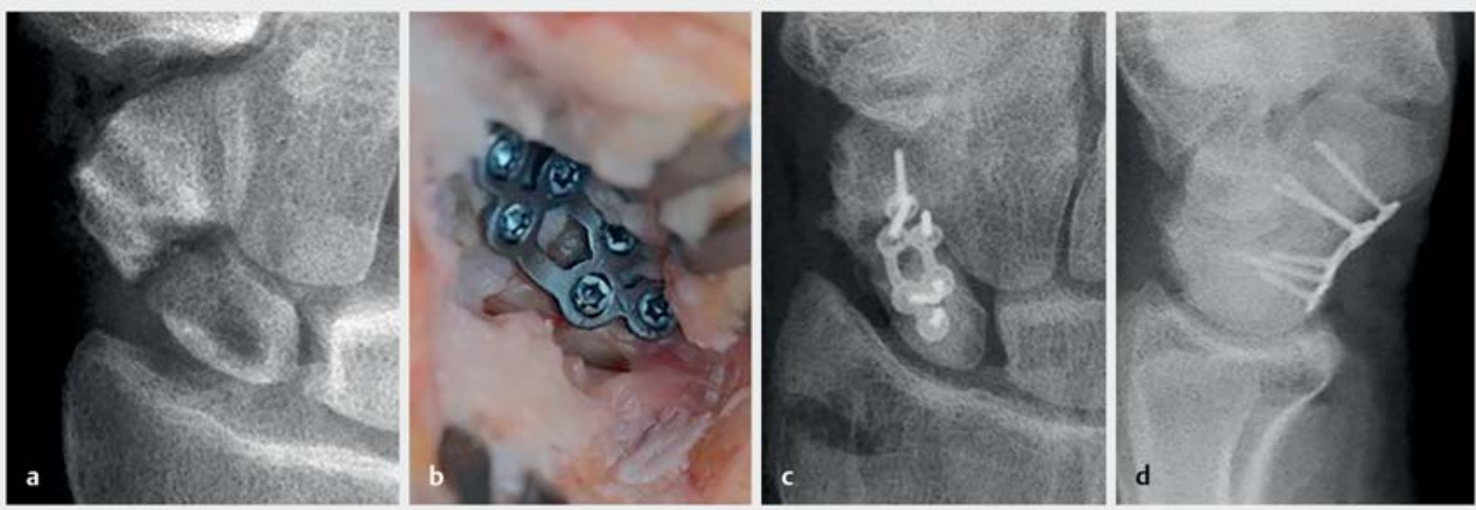

- Abb. 4 Große Defekte des Kahnbeins nach fehlgeschlagener Osteosynthese. a Skaphoid nach Implantatentfernung und Débridement. b Intraoperativer Situs nach Knochentransplantation und Schraubenosteosynthese. c, d Röntgenbilder der Plattenosteosynthese.

dioskapholunäre Fusion (RSL-Fusion) ( $\mathbf{A b b}$. 6) eine gute Lösung sein, insbesondere wenn noch zusätzlich der distale Skaphoidpol mitreseziert wird, da dadurch die Beweglichkeit im einzig verbliebenen Mediokarpalgelenk noch verbessert wird.
Ist die Fovea lunata noch von guter Qualität und das Os lunatum noch gut beweglich, dagegen aber der proximale Anteil des Os capitatum arthrotisch, ist die beste Lösung eine mediokarpale Teilarthrodese, zu der die Skaphoidresektion obligatorisch dazugehört. 

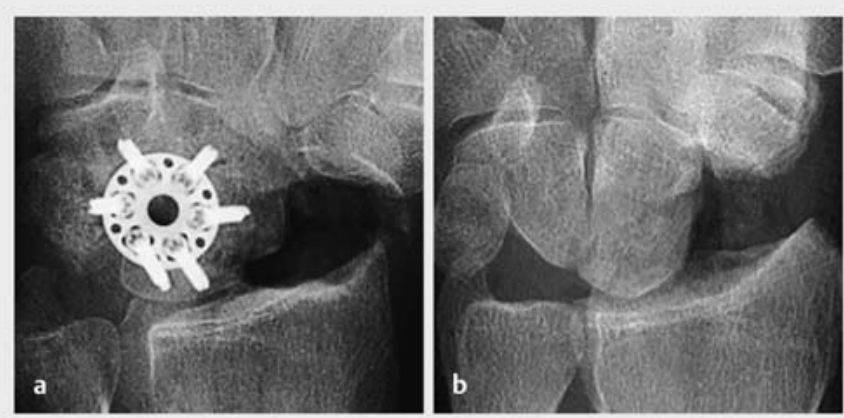

Abb. 5 Rettungsoperationen. a Entfernung des nicht rekonstruierbaren Kahnbeins und mediokarpale Teilfusion mit Plattenosteosynthese. b Entfernung der gesamten 1. Handwurzelknochenreihe (Proximal Row Carpectomy).

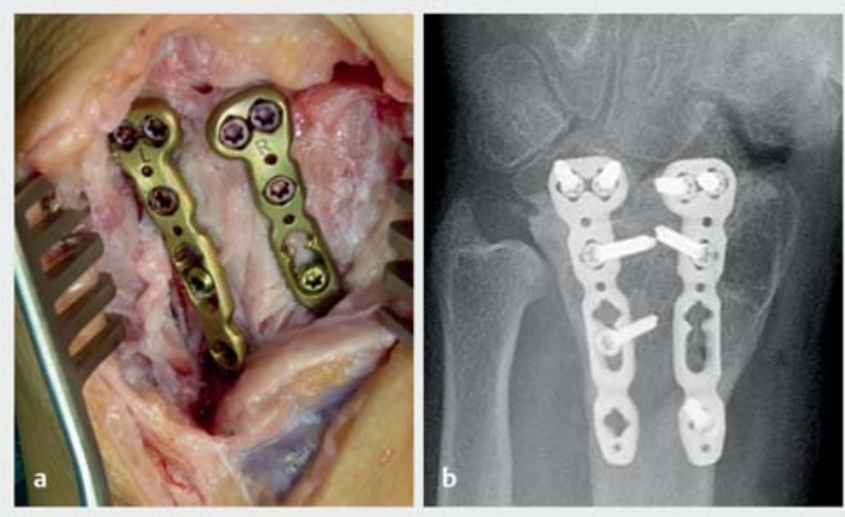

- Abb. 6 Radioskapholunäre Fusion. a Intraoperative Darstellung der Plattenarthrodese zwischen Radius, Kahnbein und Mondbein. b Röntgenbild der radiokarpalen Fusion.
Ist die Fovea lunata von guter Qualität, das Os lunatum aber in Fehlstellung und nur wenig beweglich, so hat die Entfernung der gesamten 1. Handwurzelreihe (Proximal Row Carpectomy) die besten Ergebnisse ( $\bullet$ Abb. $\mathbf{5 b}$ b).

\section{Fehlgeschlagene Osteosynthesen an anderen Handwurzelknochen}

Die Osteosynthesen an anderen einzelnen Handwurzelknochen sind insgesamt selten und müssen daher noch seltener revidiert werden. In unserer Klinik mussten in den vergangenen Jahren nur wenige revidiert werden. In einem Fall war die Schraubenosteosynthese am Os trapezium nicht ausreichend stabil genug und es kam zum Abrutschen des radialen Fragments. Möglich sind auch Fehlplatzierungen von Schrauben, z. B. bei einer Hamulusfraktur, die dann in Gelenkspalten liegen können. Weitere fehlgeschlagene Osteosynthesen im Handwurzelbereich sind gelockerte Teilarthrodesen (z. B. die mediokarpale Teilarthrodese oder STT-Arthrodese) mit Implantatbrüchen und Pseudarthrosen.

Bei diesen fehlgeschlagenen Teilarthrodesen, die noch nicht durch eine komplette Handgelenksarthrodese behandelt werden sollen, müssen die eingebrachten Implantate meist komplett entfernt, die Pseudarthrosenbereiche sauber reseziert und mit vorzugsweise reichlich Beckenkammspongiosa aufgefüllt werden, um dadurch eine stabile Osteosynthese zu erreichen. Bei den multidirektionalen Plattensystemen ist manchmal noch genug stabiler Knochen zur Neuplatzierung der Schrauben vorhanden ( $\boldsymbol{A}$ Abb. 7). Aber auch die Stabilisierung durch mehrere K-Drähte ist weiterhin eine gute Option.

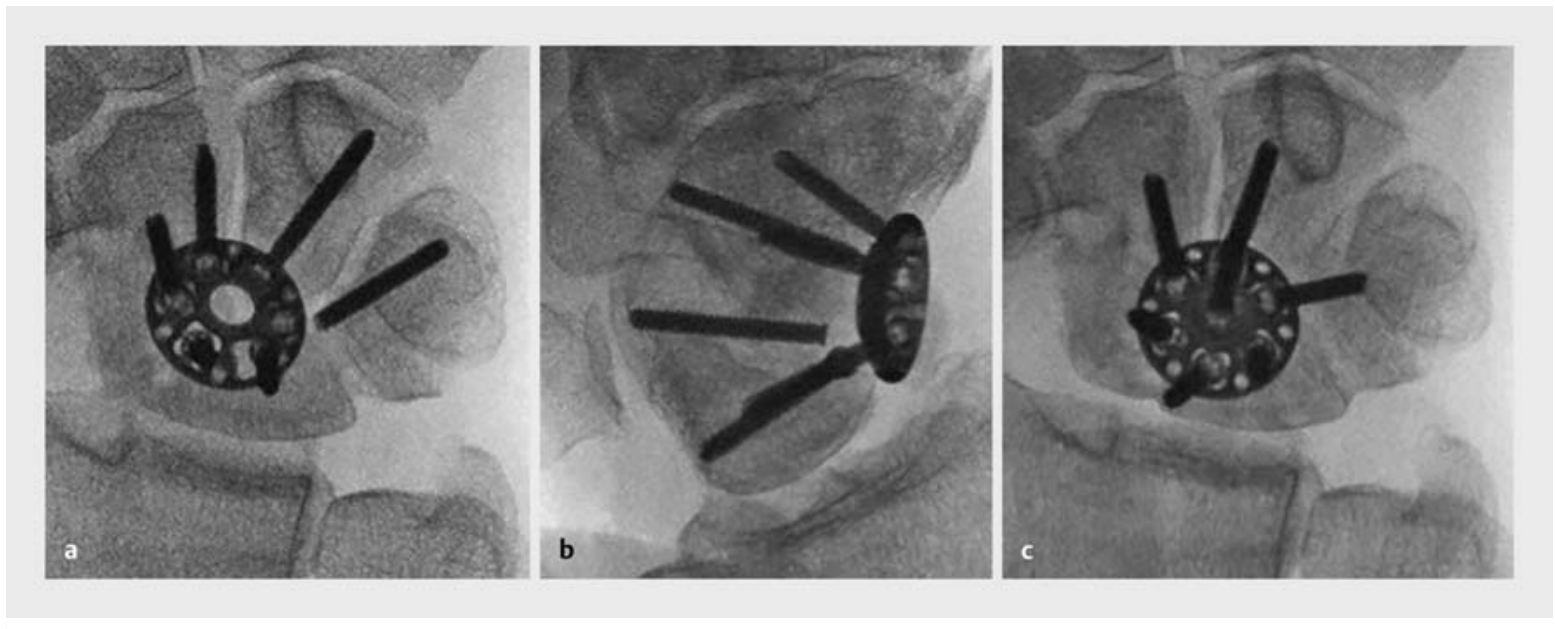

- Abb. 7 Pseudarthrose einer mediokarpalen Fusion. a, b Schraubenbrüche. c Nach Pseudarthrosenresektion, Beckenkammspongiosaauffüllung und geänderter Schraubenposition mit gleicher Platte (Carpal Fusion Plate). 


\section{Fehlgeschlagene Osteosynthesen} im Bereich der Karpometakarpalgelenke und der Metakarpalbasen

Der größte Anteil an fehlgeschlagenen Osteosynthesen in diesem Bereich entfällt auf die Arthrodesen. Die Gelenke weisen nur einen geringen Bewegungsumfang auf

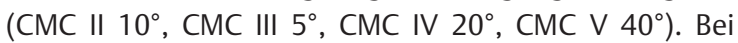
Trümmerfrakturen oder großen Instabilitäten wird hier nicht selten eine Arthrodese durchgeführt ( $\bullet$ Abb. 8). Plattenbrüche und Pseudarthrosen sind hier nicht selten, da die Beweglichkeit hier unterschätzt wird.

\section{Lösungsmöglichkeiten:}

- erneute Arthrodese mit Spongiosaplastik, Leiterplattenosteosynthese, ggf. zusätzlich K-Drähte

- Resektionsarthroplastik im CMC-5-Gelenk mit Sehneninterposition

- Dubert-Arthrodese ( $>$ Abb. 9) [4, 8-11]

\section{Fehlgeschlagene Osteosynthesen im Metakarpalschaftbereich}

Die Osteosynthesen in diesem Bereich werden durch KDrähte, intramedulläre Drähte, Schrauben, neuerdings auch durch intramedulläre Schrauben und Platten durchgeführt. Häufigstes Problem ist die Torsionsabweichung. Das Überkreuzen der Finger oder das zu weite Abstehen der äußeren Finger stört die Funktion der Hand gewaltig. Der Begriff „Torsionsabweichung“ ist im klinischen Sprachgebrauch in Anwesenheit von Patienten deutlich

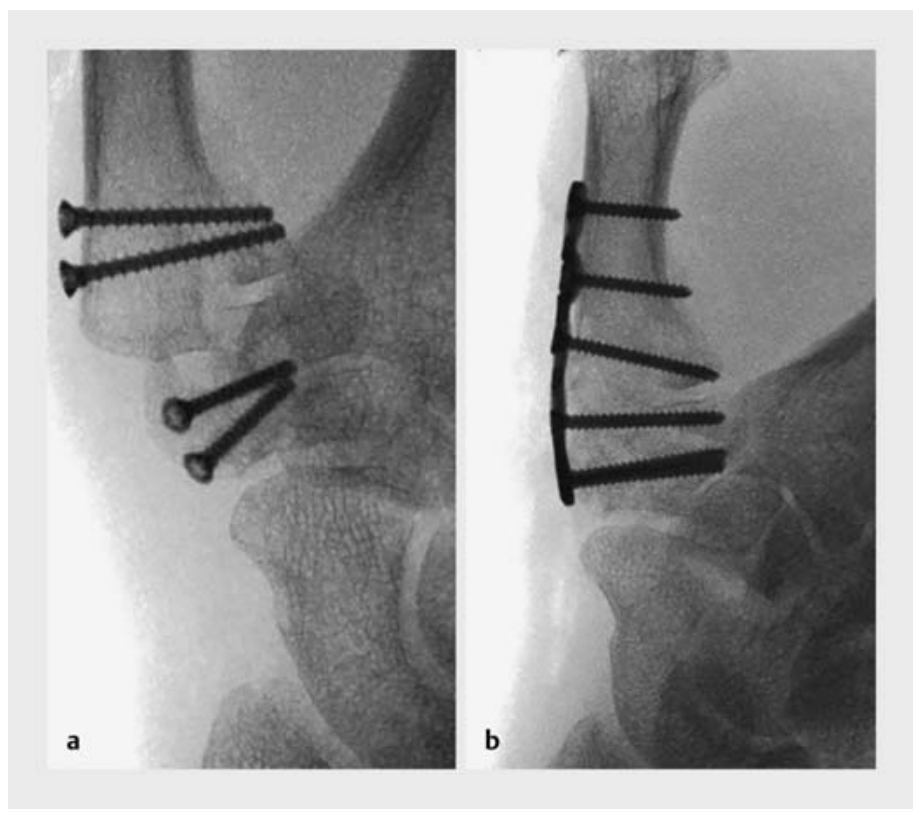

- Abb. 8 Kombinierte Bennett- und Trapeziumfraktur. a Ursprüngliche anatomische Schraubenosteosynthese hatte Instabilität der kombinierten Bennett- und Trapeziumfraktur nicht berücksichtigt: deutliches Abrutschen der Fragmente. b Bei kompletter Zerstörung der Gelenkflächen und Bandinstabilität: Arthrodese des Daumensattelgelenks mit winkelstabiler T-Plattenosteosynthese.

weniger forensisch triggernd als der Begriff „Rotationsfehler“. Daneben gibt es noch Implantatbrüche, Pseudarthrosenfehlstellungen und Verkürzungen der Knochen, die funktionelle Probleme bereiten. Auch hier sollen die häufigsten Szenarien dargestellt werden.
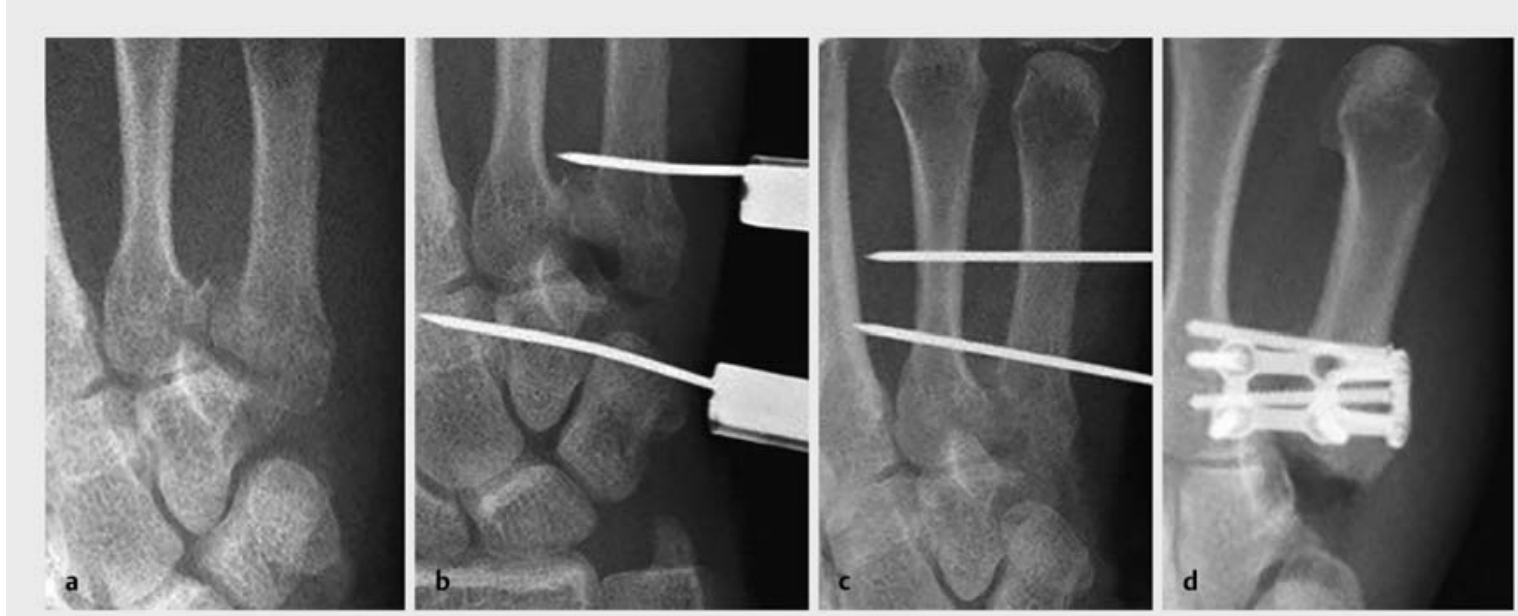

Abb. 9 Veraltete imprimierte Karpometakarpale-V-Basistrümmerfraktur. a Eingestauchte Basisfraktur des 5. Mittelhandknochens. b Intraoperativer K-Draht-Arthrodesenspreizer zum Reponieren des MC V nach distal. c K-Draht-Transfixation MC V unter Distraktion an MC IV. d Nach Implantatentfernung Krepitation und Schmerzen im CMC-V-Gelenk: Resektionsarthroplastik und Dubert-Arthrodese der Basis MC IV an Basis MC IV. 

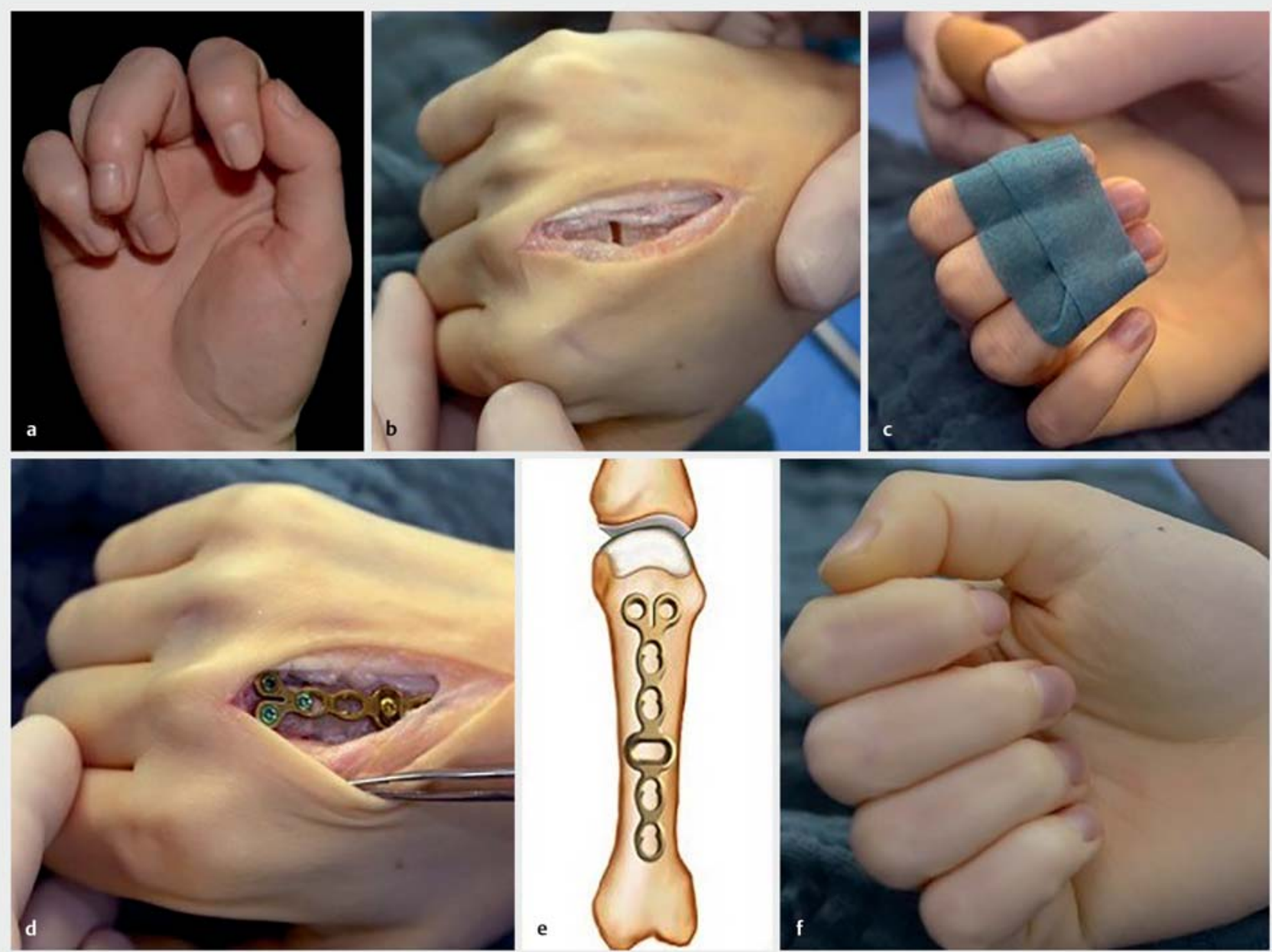

- Abb. 10 Deutliche Torsionsabweichung des Mittelfingers nach instabiler K-Draht-Transfixation einer Metakarpale-III-Fraktur von distal. a Extreme Torsionsabweichung. b Dorsale Eröffnung und Querosteotomie des Metakarpale III. c Tapefixierung der Finger DII-DIV zur Grobreposition. d Plattenosteosynthese mit der Rotation Correction Plate mit dem transversalen Gleitloch zur Feineinstellung der Torsion nach Entfernung des Tapes. e Plattenlage auf dem Metakarpale III. f Fingerstellung nach kompletter Plattenfixierung.

\section{Szenario 1: Osteosynthese mit deutlicher Torsionsabweichung}

Das Überkreuzen der Finger beim Faustschluss kann bereits bei minimalen Fragmentverdrehungen im Mittelhandbereich auftreten ( $\boldsymbol{A}$ Abb. 10). Ursache kann z.B. eine ungenügende Längenreposition bei Spiralfrakturen sein. Bei Plattenosteosynthesen am Metakarpale kann eine leicht seitlich verrutschte Bohrung die Platte und die Fragmente in eine ungewollte Position bringen [1215]. Ein Versetzen der Schrauben um 0,5 mm nach radial oder ulnar ist nicht möglich. Lösung für diese Probleme ist eine neue Plattenosteosynthese mit einer von uns entwickelten Spezialplatte, die ein transversales Gleitloch aufweist. Diese Platten verwenden wir in unserer Klinik seit 2005. Seit 2006 sind sie auch als winkelstabile Implantate erhältlich. Am leichtesten können diese Platten bei bereits (fehl-)verheilten Knochen eingesetzt werden, da hier eine Osteotomie gesetzt werden muss. Diese Os- teotomie kann man dann exakt $90^{\circ}$ zur Längsachse anlegen, was die Drehung in die optimale Position wesentlich erleichtert. Aber es ist durchaus auch gut möglich, diese Platten bei frischen Korrekturen oder sogar primär, z. B. bei Replantationen auf Mittelhandhöhe, einzusetzen. Beim Einsatz dieser Platte wird die Platte so angelegt, dass das distale oder proximale Fragment mit winkelstabilen Schrauben besetzt werden kann und das andere Fragment den Abschnitt der Platte mit dem transversalen Gleitloch enthält. Nach Entfernung der Implantate der vorausgegangenen Operation und nach Mobilisierung der alten Fraktur bzw. der Querosteotomie wird die „Correction Osteotomy Plate“ in Längsrichtung angelegt und distal mit winkelstabilen Schrauben fixiert. Die Reposition der Fraktur erfolgt zunächst grob in Faustschlussstellung der Finger. Dann wird eine Kortikalisschraube in das transversale Gleitloch in das proximale Fragment eingebracht und mäßig fest gezogen. Dann wird die Fingerstel- 
lung in Streckung und Beugung mehrfach überprüft. Durch Lösen und Festziehen der Kortikalisschraube im transversalen Gleitloch kann die Torsion des Fingers stufenlos eingestellt werden, bis die optimale Position erreicht ist. Dann werden die anderen proximalen Schrauben gebohrt und besetzt.

\section{Szenario 2: Implantatbruch oder Auslockerung mit Pseudarthrosenbildung}

Bei einem solchen Szenario findet sich reichlich Narben-, Pseudarthrosen- und Synovialitisgewebe im ehemaligen Frakturbereich, das sauber entfernt werden sollte. Das gebrochene Implantat muss ebenfalls entfernt werden. Wenn die Schrauben sowohl proximal als auch distal fest gesessen haben, kann man sich überlegen das exakt gleiche neue Implantat wieder aufzubringen, den Pseudarthrosenbereich mit Beckenkammspongiosa aufzufüllen und einen Mittelhandbrace für 4 Wochen zu verordnen. Eine größere Stabilität erhält man immer mit einer Leiterplatte, um die Torsion exakt einzustellen, ist aber auch hier eine Rotation Correction Plate möglich.

\section{Szenario 3: Osteosynthese in deutlicher Verkürzung}

Eine Verkürzung eines Mittelhandknochens kann auftreten, wenn bspw. eine eingestauchte Halsfraktur durch eine intramedulläre Osteosynthese versorgt wurde und mehrere Versuche notwendig waren, der Kopf dann aber über den Drähten wieder nach proximal sinkt, oder wenn eingestauchte Frakturen durch retrograde K-Drähte (oder durch Platten) ohne Wiederherstellung der Länge fixiert wurden. Eine deutliche Verkürzung der Mittelhandknochen hinterlässt einen deutlichen Spannungs- und Kraftverlust der Streck- und Beugesehnen. Ein Streck- und Beugedefizit sind die Folge. Die Patienten profitieren von der Wiederherstellung der Länge sehr. Um die Länge zu rekonstruieren, ist neben einer stabilen Plattenosteosynthese - auch hier bevorzugen wir wegen der exakten Einstellbarkeit der Torsion die Rotation Correction Plate, denn der Patient erwartet bei einer Revisionsoperation ein in jeder Hinsicht perfektes Ergebnis - eine Interposition eines stabilen Beckenkammspans notwendig. Um die verkürzten Weichteile wieder zu verlängern, ist neben der Narbenresektion häufig eine quere Periostinzision und eine Dehnung der Weichteile durch Einbringen und maximales Aufspreizen eines Arthrodesenspreizers für einige Minuten im Frakturspalt hilfreich.

\section{Fehlgeschlagene Osteosynthesen im Metakarpalehals- und -kopfbereich}

Osteosynthesen mit Metakarpalekopfbereich sind bei intraartikulären Frakturen notwendig. Aufgrund der großen, fast semizirkulär umfassenden Knorpeloberfläche des Grundgliedkopfes beeinträchtigen viele Osteosyn- thesen die Kapsel oder die Knorpelflächen. Folgen davon sind deutliche Beugedefizite im Grundgelenk.

Am häufigsten ist der Halsbereich des Metakarpale $V$ betroffen. Diese Frakturen lassen sich i.d.R. hervorragend durch intramedulläre Drähte stabilisieren (Küntscher/ Foucher) $[9,10,16]$.

Bei unterschätzten Mehrfragmentbrüchen kann es zum Auswandern der Drähte kommen. Die Revisionsoperation gelingt am besten mit einer winkelstabilen Plattenosteosynthese mit weit nach distal reichendem flachen Schraubenverlauf, um hier außerhalb der Knorpelflächen zu bleiben ( $\triangleright$ Abb. 11) (Metacarpal Neck Plate, die von uns 2009 entwickelt wurde). Normale Plattenosteosynthesen liegen meist dem Knorpel auf und führen zu starken Bewegungseinschränkungen.

Als Rettungsoperation im Metkarpalkopfbereich stellt die Grundgelenksendoprothetik eine häufig sinnvolle Lösung dar.

\section{Fehlgeschlagene Osteosynthesen im Grundgliedbasisbereich}

Fehlgeschlagene Osteosynthesen im Grundgliedbasisbereich sind verbliebene, nicht erkannte oder sekundär entstandene Gelenkstufen sowie seitliche Frakturen mit nicht ausreichend reponierten Ausrissfragmenten der Kollateralbänder. Die Gelenkstufen sollten über einen dorsalen Zugang unter Sicht reponiert werden. Schwierig ist dann die Stabilisierung, denn die Fragmente sind häufig sehr klein und der Zug der Kollateralbänder kräftig, sodass wenig Platz für Repositionszangen oder andere Hilfsmittel bleibt. Individuell angefertigte Krallenplatten oder retrograde Verschraubungen können hier sinnvoll eingesetzt werden [17].

\section{Fehlgeschlagene Osteosynthesen im Grundgliedschaftbereich}

Grundgliedschaftfrakturen sind häufig und viele lassen sich erstaunlich gut konservativ behandeln, kombiniert mit frühzeitiger Übungsbehandlung. Auch wenn häufig keine anatomische Heilung durch die konservative Therapie erreicht wird, ist doch die Beweglichkeit der Fingergelenke und die Funktion der Hand normal, was man bei vielen Osteosynthesen mit exakter anatomischer Reposition nicht behaupten kann. Nicht alle Frakturen lassen sich konservativ behandeln und die häufigsten Osteosynthesen sind K-Draht-Fixationen, Schraubenosteosynthesen und Plattenosteosynthesen. Gewindedrähte oder intramedulläre Herbert-Schrauben sind noch neu und noch nicht verbreitet. 

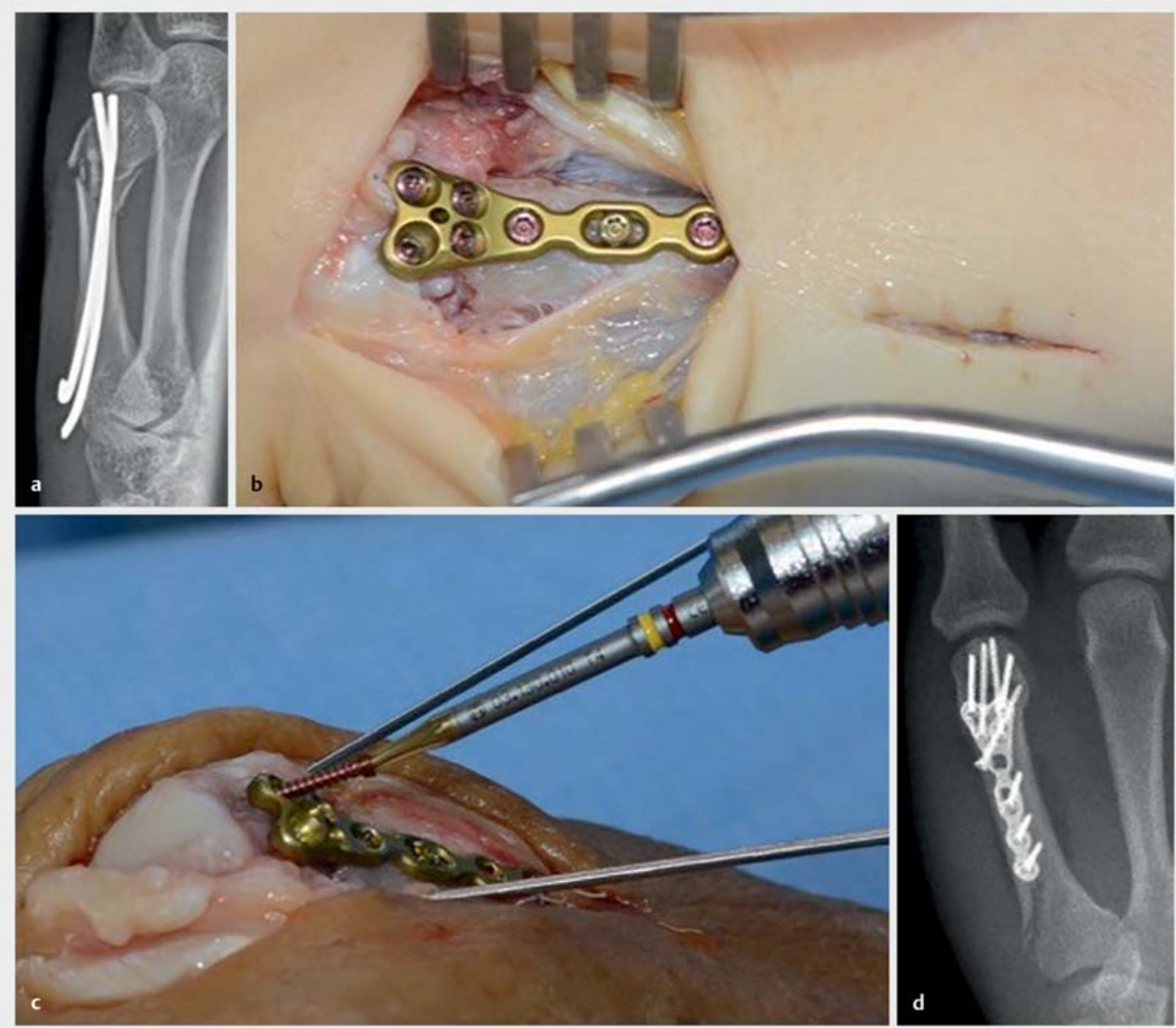

- Abb. 11 Ausgebrochene intramedulläre Drähte bei Metkarpale-V-Hals- und -kopftrümmerfraktur. a Distal ausgebrochene Drähte mit Perforation in das Grundgelenk. b Entfernung der intramedullären Drähte und Anbringen der winkelstabilen Metacarpal Neck Plate. c Stabilisierung des Mittehandknochenkopfes durch extrem flach einschraubbare winkelstabile Schrauben, sodass die Platte außerhalb des Knorpelbereichs zu liegen kommt. d Röntgenbild nach der Reposition des mehrfragmentären Metakarpalkopfes und Metakarpalhalses.

Osteosynthesen im Grundgliedschaftbereich können folgende Fehlschläge aufweisen [18]: Torsionsabweichungen, Achsfehler zur Seite oder in sagittaler Richtung, zu lange Schrauben, die in die Beugesehnen reichen und diese blockieren, Drahtlockerungen, Schraubenausbrüche oder Plattenbrüche mit Pseudarthrosen, vor allem aber die Verklebung der Streckaponeurose mit dem Grundgliedknochen, was zu enormen Bewegungseinschränkungen der Fingergelenke führt [19].

Auch hier die wichtigsten Szenarien:

\section{Szenario 1: Schrauben zu lang in der Beugesehne}

Wenn es auffällt, dass die Schrauben zu lang sind und die Beugesehnen blockieren, sollte kurzfristig operiert werden. Gegebenenfalls reicht es, die Platte zu belassen und nur die Schrauben zu kürzen - häufig sind monokortikale Schrauben im Grundgliedschaftbereich vollkommen ausreichend. Zusätzlich sollte aber auch palmar eröffnet werden und eine Tenolyse der Beugesehnen vorgenommen werden. Zu lange Schrauben bewirken im Bereich der Irritation eine Auffaserung der Sehne, eine starke Entwicklung von Synovial- und Narbengewebe zwischen Beugesehnen und Knochen. Bleibt dies längere Zeit bestehen, entwickelt sich eine feste, langstreckige Narbenplatte, die nur operativ gelöst werden kann.

\section{Szenario 2: Torsionsabweichung}

Torsionsabweichungen im Grundgliedbereich sind tückisch, da durch die Korrektur und Revisionsoperation eine hohe Verklebungsgefahr der Streckaponeurose mit dem Knochen besteht. Bei kleineren Abweichungen kann anstatt auf Grundgliedhöhe eine Korrektur auf Höhe der 


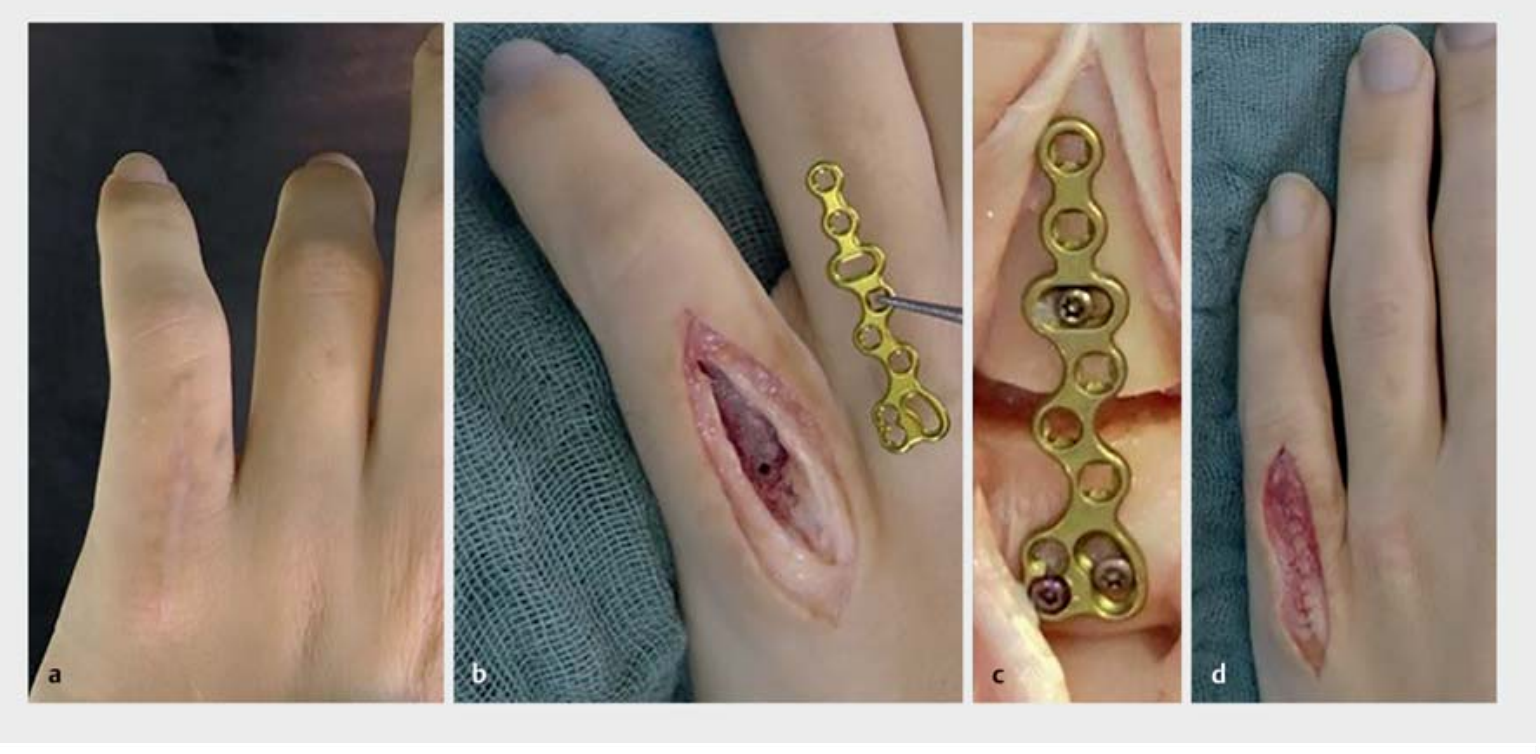

- Abb. 12 Kombinierte Achs- und Torsionsfehlstellung des Grundglieds nach dorsaler Plattenosteosynthese. a Fingerstellung präoperativ. b Entfernung der primär eingebrachten Platte und Anhalten der „Banana Hole Fracture Plate“. c Breite proximale Querosteotomie und Besetzen der Platte mit einer winkelstabilen Schraube proximal und je einer Kortikalisschraube in das bananenförmige Langloch und das distale transversale Langloch. Jetzt kann jede Kombination einer Torsions- und Achsabweichung fein eingestellt werden. In optimaler Position werden die übrigen Schrauben besetzt.

Mittelhandknochen (extrafokal) sinnvoll sein. Die Komplikationsrate ist im Mittelhandknochenbereich deutlich geringer. Größere Torsionsabweichungen müssen dagegen intrafokal korrigiert werden. Bis etwa zur 10 . Woche nach der Fraktur können die eingebrachten Implantate und auch noch der gebildete Kallus entfernt werden und somit anatomische Nachrepositionen möglich sein. Nach der 10 . Woche muss i.d. R. osteotomiert werden. Intrafokale Osteotomien sind am günstigsten, da die ursprüngliche Anatomie am besten ohne Translationen wiederhergestellt werden kann. Bei Querosteotomien muss auf die Beugesehnenscheide (A2-Ringband) geachtet werden, die durch die Drehosteotomie nicht eingeengt werden darf. In diesen Fällen ist eine diagonale Osteotomie mit sehr schmaler dorsaler Keilresektion wesentlich günstiger, aber auch wesentlich anspruchsvoller.

Je nach Situation muss mit K-Drähten, Schrauben oder Platten gearbeitet werden. Wir haben gute Erfahrungen mit der Rotation Correction Plate gemacht, die so stabil ist, dass mit sofortigen Übungsbewegungen - noch am Operationstag - begonnen werden kann.

\section{Szenario 3: Achsabweichung}

Achsabweichungen im Grundgliedbereich müssen intrafokal korrigiert werden. Das eingebrachte Osteosynthesematerial kann meist nicht mehr genutzt werden und muss entfernt werden. Da i.d.R. gleichzeitig Verkürzungen der Knochen bestehen, sind eröffnende (open wedge) Osteotomien notwendig, die mit Spongiosa aufgefüllt werden sollten. Auch hier muss die Stabilität der
Osteosynthese so groß sein, dass sofort nach der Operation mit Bewegungsübungen begonnen werden kann. Die Osteosynthese durch K-Drähte ist hier sehr anspruchsvoll, leichter sind Leiterplattenosteosynthesen, die aber sehr zu Verklebungen der Streckaponeurosen neigen. Hier hilft nur die Periost-Gleitgewebe-Lappenplastik (Langer 1998) [20] und sofortige aktive und passive Beübung den angrenzenden Gelenke.

\section{Szenario 4: Torsions- und Achsabweichung}

Da in vielen Fällen keine reine Torsionsabweichung oder keine reine Achsabweichung nach dorsal oder palmar besteht, sondern kombinierte Fehlstellungen, sind die Korrekturen schwer zu planen. Wird bei diesen Fehlstellungen nur eine Richtung korrigiert, so kann sich eine andere Richtung verstärken. So kann sich bspw. bei Korrektur der Rotation eine Achsabweichung deutlicher darstellen oder bei einer Korrektur der Achse plötzlich ein Überkreuzen der Finger einstellen. Um dies zu korrigieren, ist die von uns 2007 entwickelte „Banana-Hole“-Korrekturplatte ideal ( $\triangleright$ Abb. 12). Hier ist es bei liegender Platte möglich, sowohl die Torsion als auch die Achse stufenlos zu korrigieren und vor allem intraoperativ so lange nachzureponieren, bis die optimale Stellung erreicht ist.

\section{Szenario 5: Plattenbruch}

Die Ursache für einen Plattenbruch im Grundgliedbereich ist eine verbleibende Instabilität trotz Osteosynthese während der notwendigen Übungsbehandlung. Ein nicht gefasstes keilförmiges Fragment, ein Knochendefekt unter einer dünnen Platte, insbesondere wenn über dem 
Knochendefekt ein unbesetztes Plattenloch liegt, und vieles mehr sind mögliche Ursachen, die genau analysiert werden müssen. Die gebrochene Platte muss entfernt werden, das Narben-/Pseudarthrosengewebe sauber entfernt und ggf. mit Spongiosa aufgefüllt werden. Meist ist dann eine Leiterplattenosteosynthese notwendig.

\section{Szenario 6: Verklebung der Streckaponeurose nach Plattenosteosynthese}

Die Verklebungen der Streckaponeurose über dem Grundglied sind die häufigsten Fehlschläge einer Plattenosteosynthese oder wenn K-Drähte die Streckaponeurose direkt am Knochen fixieren. Da der Knochen nach meist 3 bis 4 Wochen verheilt ist, kann eine frühe Implantatentfernung und Tenolyse geplant werden. Bei der Physiotherapie sollte das Mittelgelenk nicht mit allzu großer Kraft gebeugt werden, um die Streckaponeurose doch noch zu mobilisieren. Das Ergebnis ist dann eher ein Ausleiern des Strecksehnenmittelzügels als eine Mobilisierung der Streckaponeurose. Die meisten Platten wurden streckseitig über eine Spaltung der Streckaponeurose in der Medianlinie eingebracht. Der gleiche Zugangsweg wird wieder eröffnet, die Streckaponeurose wieder in der Medianlinie gespalten und das Implantat entfernt. Mit einem kleinen Luer wird das Implantatlager gesäubert und dann die Streckaponeurose nach proximal und distal gelöst. Am Knochen sollte eine dünne Schicht Periost belassen werden, was sehr diffizil und schwierig ist und was bei Weitem nicht immer gelingt [20]. Das Ausmaß der Tenolyse richtet sich nach dem Ausmaß der Verklebungen. Die Tenolyse muss so weit fortschreiten, dass das PIP-Gelenk (PIP: proximales Interphalagealgelenk) und das Grundgelenk passiv frei bis $90^{\circ}$ (Grundgelenk) und über $100^{\circ}$ (PIP-Gelenk) beugbar sind. Dies wird häufig nur dadurch erreicht, dass die gesamte Streckaponeurose, und hier insbesondere auch die wichtigen Seitenzügel, langstreckig von den Verklebungen gelöst werden. Ein Zug mit der Pinzette an der Streckaponeurose über dem Grundgelenk muss das PIP bis zu etwa $20^{\circ}$ (verbliebener Beugung) strecken können und ein Zug an den Seitenzügeln nahe der Interosseusmuskulatur das PIP-Gelenk vollständig strecken können.

\section{Fehlgeschlagene Osteosynthesen im Grundgliedkopfbereich}

Grundgliedkopffrakturen sind meist Kondylenfrakturen, die sehr genau anatomisch rekonstruiert werden müssen [21]. Schon kleine Verschiebungen führen zu Gelenkproblemen mit Achsabweichungen. Der Zeitpunkt der Revision muss sorgfältig ausgesucht werden. Eine frühzeitige Revision kann dazu führen, dass die Fragmente noch sehr weich sind und zerfallen oder zerbrechen können, kein Raum für Neuplatzierungen von Drähten oder Schrauben vorhanden ist und die Gelenke durch die erneute Operation so anschwellen, dass die Beweglichkeit trotz Hand- therapie und anatomischer Reposition schlecht wird [22]. Wird dagegen die Fehlstellung erst akzeptiert zugunsten einer intensiven Übungsbehandlung, kann eine Revision an einem gut beweglichen Gelenk als Korrekturosteotomie mit festen Knochenverhältnissen erfolgen. Nach der Korrekturosteotomie kann dann die Übungsbehandlung mit einem größeren Bewegungsumfang erfolgen.

\section{Fehlgeschlagene Osteosynthesen im Mittelgliedbasisbereich}

Der Mittelgliedbasisbereich ist ein sehr spezieller Bereich [23-26]. Der dorsale Abschnitt beinhaltet den Ansatz des Mittelzügels der Streckaponeurose. Nicht reponierte Fragmente können daher zur Knopflochdeformität führen. Der palmare Abschnitt des Mittelgelenks beinhaltet den Ansatz der palmaren Platte, der sehr häufig bei den Überstreckverletzungen der Mittelgelenke abreißt. Diese Verletzungen brauchen meist nur reponiert und kurzzeitig in Streckstellung geschient zu werden. Eine sehr ähnlich aussehende Fraktur der palmaren Mittelgliedbasis kann aber schnell in einer Katastrophe enden: der Mittelgliedbasisimpressionsfraktur. Der Verletzungsmechanismus ist keine Überstreckung, sondern eine axiale Kompressionsfraktur bei leicht gebeugtem Finger. Der dorsale Abschnitt der Mittelgliedbasis luxiert nach dorsal, der zentrale Anteil ist eingestaucht und der palmare Abschnitt ebenfalls eingestaucht und palmar verlagert. Die Gelenkfläche ist instabil und Sehnen und Bänder gestaucht im Ungleichgewicht. Dies führt unbehandelt zu einer Arthrose und Gelenkeinsteifung. Die Operation dieser imprimierten und dislozierten Gelenkanteile ist sehr anspruchsvoll und birgt zahlreiche Möglichkeiten für Fehlschläge. In den letzten Jahren haben die dynamischen Bewegungsfixateure, von denen der Suzuki-Fixateur aus K-Drähten und Gummiringen der bekannteste ist, stark an Popularität gewonnen. Auch hier gibt es zahlreiche Fehlermöglichkeiten. Entscheidend für die Art der Revision ist der Zeitraum zwischen der operativen Versorgung und der Problemerkennung. Hier ist ein individuelles Vorgehen angezeigt und meist noch aufwendiger und schwieriger als bei der Erstoperation.

Möglichkeiten der Revision:

- dynamischer Distraktions-Bewegungsfixateur mit Zurückstößelung der imprimierten Gelenkanteile nach Hintringer

- Ersatz der zerstörten palmaren Mittelgliedbasis durch ein Knorpel-Knochen-Transplantat aus dem Os hamatum (Hemihamatum) 


\section{Fehlgeschlagene Osteosynthesen im Mittelglied}

Fehlschläge in Mittelgliedschaft- und -kopfbereich entsprechen weitestgehend denen der Grundglieder. Meist sind hier dorsale Plattenosteosynthesen aufgrund der Enge zwischen Streckaponeurose und Knochen nicht möglich oder führen in fast allen Fällen zu einer Unbeweglichkeit der Streckaponeurose mit funktioneller Einsteifung des Endgelenks in Streckstellung. Durch forcierte Übungen leiert dann der Streckapparat aus und es resultiert ein hängendes Endglied, das nur schwer zu behandeln ist.

\section{Fehlgeschlagene Osteosynthesen im Endgliedbasisbereich}

Der dorsale Endgliedbasisabschnitt ist sehr häufig von Frakturen betroffen (Segond-Busch-Fraktur). Verletzungsmechanismus ist fast nie ein Hyperflexionstrauma, was der häufig verwendete Ausdruck „Strecksehnenabriss“ suggeriert, sondern meist ein HyperextensionsKompressions-Mechanismus. Das hängende Endglied stört viele Patienten sehr. Wenn das Fragment nicht zu sehr disloziert ist und keine palmare Luxation des Endglieds vorliegt, können sehr gute Ergebnisse mit einer konservativen Therapie erreicht werden. Falls doch operiert werden muss, bestehen zahlreiche Komplikationsmöglichkeiten: Das Fragment kann nicht richtig reponiert sein, zerbersten, die K-Drähte können den Knorpel extrem schädigen, Anker verrutschen, Hautprobleme entstehen etc. etc. Als Rettungsoperation verwenden wir die minimalinvasive transossäre PDS-Cerclage-Technik (PDS: resorbierbarer monofiler Faden), mit der kleine oder zerbrochene Fragmente gut reponiert werden können.

\section{Sonstige fehlgeschlagene Osteosynthesen im Endgliedbereich}

Nahezu alle Osteosynthesen im Endgliedbereich werden durch Drähte, feine Schrauben und maximal bei Endgelenksarthrodesen durch Herbert-Schrauben versorgt. Komplikationen sind hier Ausbrechen oder Lockerungen dieser Implantate. Revisionsoperationen sind aufgrund der Kleinheit der übrig gebliebenen Fragmente und aufgrund der begrenzten Zugangswege (Nagelbett und Fingerkuppe) aufwendig und diffizil.

\section{Interessenkonflikt}

Die Autorinnen/Autoren geben an, dass kein Interessenkonflikt besteht.

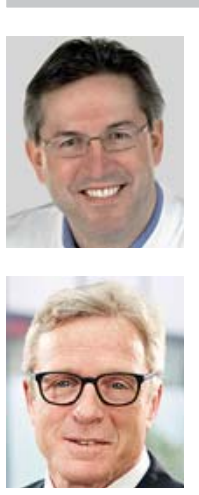

\section{Martin F. Langer}

Prof. Dr. med. Leiter der Sektion Handchirurgie und Mikrochirurgie. Klinik für Unfall-, Handund Wiederherstellungschirurgie, Universitätsklinikum Münster

\section{Michael J. Raschke}

Prof. Dr. med. Direktor der Klinik. Klinik für Unfall-, Hand- und Wiederherstellungschirurgie, Universitätsklinikum Münster

Korrespondenzadresse

Prof. Dr. med. Martin Franz Langer

Klinik für Unfall-, Hand- und Wiederherstellungschirurgie Universitätsklinikum Münster

Waldeyerstr. 1

48149 Münster

Tel.: $0251 / 8356337$

Fax: 0251/8356318

Martin.Langer@ukmuenster.de

\section{Literatur}

[1] Langer MF, Unglaub F, Breiter S et al. Anatomie und Pathobiomechanik des Skaphoids. Unfallchirurg 2019; 122: 170-181

[2] Fernandez DL. The author's technique for the management of unstable scaphoid nonunions. Hand Clin 2019; 35: 271-279

[3] Yeo JH, Kim JY. Surgical strategy for scaphoid nonunion treatment. J Hand Surg (Asian-Pacific Volume) 2018; 23: 450-462

[4] Jurkowitsch J, Dall'Ara E, Quadlbauer $S$ et al. Rotational stability in screw-fixed scaphoid fractures compared to plate-fixed scaphoid fractures. Arch Orthop Trauma Surg 2016; 136: 1623-1628

[5] Kalb KH, Langer MF, Windolf J et al. Skaphoidpseudarthrose. Komplexe Rekonstruktionen mithilfe vaskularisierter Knochentransplantate. Unfallchirurg 2019; 122: 200-210

[6] Boe CC, Amadio PC, Kakar S. The management of the healed scaphoid malunion. What to do? Hand Clin 2019; 35: 373-379

[7] Dubert T. Arthroplastie stabilisée du cinquième métacarpien. Proposition thérapeutique pour le traitement des fractures-luxations anciennes de 5e métacarpien. Ann Chir Main 1994; 13: 363-365

[8] Förstner H. Die intramedulläre Nagelung distaler Mittelhandbrüche-Technik, Instrumentarium, Fallbeispiele. Handchir Mikrochir Plast Chir 1994; 26: 29-34

[9] Foucher G, Chemorin C, Sibilly A. Nouveau procédé d'osteosynthèse original dans les fractures du tiers distal du cinquième métacarpien. Nouv Presse méd 1976; 5: 1139-1140

[10] Foucher G. "Bouquet" osteosynthesis in metacarpal neck fractures: a series of 66 patients. J Hand Surg. Am 1995; 20: $86-90$

[11] Spies CK, Langer MF, Hohendorff B et al. Offene Reposition und Schrauben-/Plattenosteosynthese von Mittelhandfrakturen. Oper Orthop Traumatol 2019; 31: 422-432

[12] Freeland AE, Lindley SG. Malunions of the finger metacarpals and phalanges. Hand Clin 2006; 22: 341-355 
[13] Gollamudi S, Jones WA. Corrective osteotomy of malunited fractures of phalanges and metacarpals. J Hand Surg $\mathrm{Br} 2000$; 25: 439-441

[14] Jawa A, Zucchini M, Lauri G et al. Modified step-cut oste- otomy for metacarpal and phalangeal rotational malunion. J Hand Surg Am 2009; 34: 335-340

[15] Weckesser EC. Rotational osteotomy of the metacarpal for overlapping fingers. J Bone Joint Surg 1965; 47A: 751-756

[16] Küntscher G. Praxis der Marknagelung. Stuttgart: Schattauer; 1962

[17] Spies CK, Langer MF, Löw S et al. Der Gelenkersatz der Fingergrundgelenke. Orthopäde 2019; 31: 386-393

[18] Unglaub F, Langer MF, Unglaub JM et al. Korrekturosteotomien an der Hand. Unfallchirurg 2018, 121: 657-668

[19] Büchler U, Gupta A, Ruf S. Corrective osteotomy for post-traumatic malunion of the phalanges in the hand. J Hand Surg Br 1996; $21: 33-42$

[20] Langer MF. Bedeutung des dorsolateralen Periost-Gleitgewebelappens bei der Plattenosteosynthese von Grundgliedfrakturen. 47. Kongress der Deutschen Gesellschaft für Handchirurgie Heidelberg 19.-21.10.2006. Heidelberg: Deutsche Gesellschaft für Handchirurgie; 2006
[21] Teoh LC, Yong FC, Chong KC. Condylar advancement osteotomy for correcting condylar malunion of the finger. J Hand Surg Br 2002; 27: 31-35

[22] Langer MF, Oeckenpöhler S, Spies CK et al. Die Arthrodese des Daumengrundgelenks durch Plattenosteosynthese. Oper Orthop Traumatol 2019. doi:10.1007/s00064-019-0592-x

[23] Langer MF, Surke C, Raschke M] et al. Palmare Mittelgliedimpressionsfraktur des Fingers. Trauma Berufskrankh 2009; 11: 366-370

[24] Unglaub F, Langer MF, Hahn P et al. Fingermittelgelenkfrakturen. Unfallchirurg 2016; 119: 133-143

[25] Unglaub F, Langer MF, Löw S et al. Offene Reposition und Schrauben-/Plattenosteosynthese von Fingergrundgliedfrakturen. Oper Orthop Traumatol 2019; 31: 408-421

[26] Vahey JW, Wegner DA, Hastings $H 3^{\text {rd }}$. Effect of proximal phalangeal fracture deformity on extensor tendon function. J Hand Surg Am 1998; 23: 673-681

\section{Bibliografie}

DOI https://doi.org/10.1055/a-1056-7275

online publiziert 04.02.2020 | OP-JOURNAL 2020; 36: 140152 @ Georg Thieme Verlag KG Stuttgart · New York ISSN 0178-1715 